\title{
Estrogens and gonadal function in schizophrenia and related psychoses
}

\author{
Anita Riecher-Rössler ${ }^{1}$, Jayashri Kulkarni ${ }^{2}$
}

\begin{abstract}
${ }^{1}$ Psychiatric University Clinics Basel, University Psychiatric Outpatient Department, c/o University Hospital Basel, Petersgraben 4, CH-4031 Basel, Switzerland; E-mail: anita.riecher@upkbs.ch

Monash Alfred Psychiatry Research Centre, The Alfred Hospital and School of Psychology and Psychiatry, Monash University - Commercial Road, Melbourne 3004, Australia; E-mail: jayashri.kulkarni@med.monash.edu.au
\end{abstract}




\section{Table of Contents}

Estrogens and gonadal function in schizophrenia and related psychoses ........ 1

$1 \quad$ Introduction ................................................................................ 4

2 Estrogens: a protective factor in schizophrenia and related psychoses?

(The estrogen protection hypothesis) ......................................................... 4

$2.1 \quad$ Historical findings .................................................................. 4

$2.2 \quad$ Basic research findings ........................................................... 4

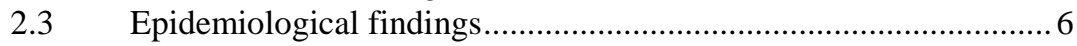

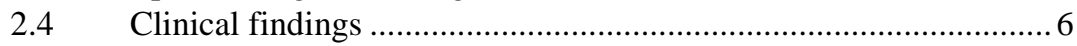

2.5 Intervention studies ............................................................... 8

3 Hypo-estrogenism in women with schizophrenia and related psychoses (The hypothesis of hypoestrogenism) ........................................................ 11

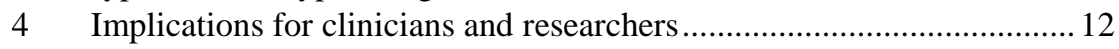

4.1 Assessment and therapy of gonadal dysfunction ......................... 12

4.2 Estradiol as a therapeutic agent?.............................................. 13

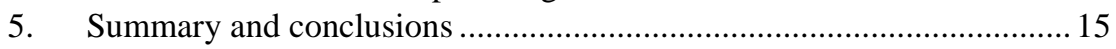

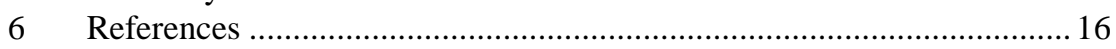


Abstract Recent research has increasingly pointed to the importance of estrogens and the hypothalamic-pituitary-gonadal axis in schizophrenia. Specifically, there is mounting evidence from clinical, epidemiological and basic research that estradiol, the main component of estrogens, exerts protective effects in schizophrenia and related psychoses. Possible modes of action of this hormone in the brain have been suggested, and clinical intervention studies have reported the first positive results. Furthermore, there are an increasing number of reports on gonadal dysfunction and states of estrogen deficiency in women with schizophrenia. These findings could have important implications for clinicians and researchers alike.

Keywords: estrogens, estradiol, schizophrenia, psychoses, gonadal function 


\section{Introduction}

Recent research increasingly points to the importance of estrogens and the hypothalamic-pituitary-gonadal axis in schizophrenia and related psychoses.

On the one hand there are reports of gonadal dysfunction and states of estrogen deficiency in women with schizophrenia (the hypothesis of hypoestrogenism). On the other hand there is mounting evidence from clinical as well as from epidemiological and basic research that estradiol, the main component of estrogens, exerts protective effects in schizophrenia and related psychoses (the estrogen protection hypothesis) [1].

\section{Estrogens: a protective factor in schizophrenia and related psychoses? (The "Estrogen Protection Hypothesis")}

\subsection{Historical findings}

As long ago as at the beginning of the last century, psychiatrists recognized the possible association between schizophrenia and estrogens (for review see [1]). There are longstanding observations indicating an association between lowered estrogen blood levels and acute psychotic symptomatology. Early clinicians such as Kraepelin and Kretschmer described signs of chronic "hypoestrogenism" in women with schizophrenia.

Kraft-Ebing was among the first to describe women becoming psychotic before or during menstruation, i.e. when blood levels of estrogen are relatively low. Kraepelin even created a separate diagnostic category, labelled "menstrual psychosis”. Kretschmer reported cases where the outbreak of schizophrenia and related psychoses had a temporal relationship with "surgery of ovaries, pregnancy, delivery and puerperium”. Finally, Manfred Bleuler noted that late-onset schizophrenia with onset after age 40 years was much more frequent in women than in men, a finding he attributed to the "loss of ovarian function" starting at around that age (for review, see [1]).

\subsection{Basic research findings}

Important findings from basic research were the identification of estrogen receptors in the limbic system of the brain, and the observation that the effects of es- 
trogens in rodents are, in some respects, similar to those of antipsychotic medications. Furthermore, it was shown that estrogens can modulate the sensitivity and number of dopamine receptors. It was therefore hypothesized that estrogens exert their antipsychotic effects in a manner similar to that of traditional antipsychotic medications at least partly by blockade of dopaminergic transmission [1]).

We now know that estrogens, and especially 17-ß-estradiol (the natural estrogen that is most active in the brain), have many other neuroprotective and psychoprotective effects. For example, they appear to improve cerebral blood flow and glucose metabolism, promote neuronal sprouting and myelination, enhance synaptic density and plasticity, facilitate neuronal connectivity, act as antioxidants, and inhibit neuronal cell death.

Estrogens have also been shown to exert profound effects on brain differentiation during development, particularly during late gestation and the early postnatal period, and are important in normal maintenance of brain function during ageing [2-5]. In a well controlled magnetic resonance imaging study, Goldstein et al. [3] showed that normal patterns of sexual brain dimorphism (brain regions found to be structurally different in normal men and women) are disrupted in schizophrenia and related psychoses, especially in the cortex. Apart from later 'activational' effects of circulating hormones (e.g. during puberty), those investigators suggested that these early 'organizational' effects of gonadal hormones that occur during the developmental period (which is probably critical for at least some forms of schizophrenia and related psychoses) could be partly responsible for that finding.

The mechanisms of action of estrogens are now known not only to depend on the classical genomic pathway but also to involve nongenomic, rapid interactions, which explains the differing latency of effects. They clearly modulate the dopaminergic and other neurotransmitter systems that are believed to be relevant to schizophrenia and related psychoses, such as the serotonergic and glutamatergic system, but also the noradrenergic and cholinergic system (for reviews [2, 4, 6-9]. Recently it has even been suggested that 17-ß-estradiol in the brain might rather be regarded as a neurotransmitter itself than as a hormone [10].

There are at least two subtypes of estrogen receptors, namely estrogen receptor$\alpha$ and estrogen receptor- $\beta$, which are transcribed from two distinct genes [4]. Autopsy studies showed that estrogen receptor- $\alpha$ messenger RNA is expressed in discrete areas of the human brain such as the amygdala, hypothalamus, cerebral cortex and hippocampus; these areas are associated with neuroendocrine function, as well as emotion, memory and cognition [11].

Recently Weickert et al. [12] reported a variation in the endogen receptor- $\alpha$ (ESR1) gene to be associated with schizophrenia and speculated that the mechanism of this association may involve alternative gene regulation and transcript processing.

Regarding the therapeutic effect of estrogens, it must also be noted that both the numerous direct effects on the brain and indirect effects may play a role. For example, estrogens may also increase blood levels of antipsychotic drugs via their actions on liver metabolism [13]. 


\subsection{Epidemiological findings}

Epidemiological studies into sex differences in schizophrenic disorders suggest that the physiologically high estradiol production in young fertile women contributes to the later age of onset of schizophrenia in women as compared with men, to the second peak of onsets in women around the menopause, and to the better course of the disease in young women [14-15]. Thus, in an epidemiological study on a representative sample of 392 first admitted patients with schizophrenia, the ABC Study, we found that schizophrenic women have a later peak of illness onset in comparison with schizophrenic men [16-18]. They also exhibit an additional, smaller peak after age 45 . We postulated that estrogens raise the vulnerability threshold for the outbreak of the disease. According to this hypothesis, women would be protected against schizophrenia between puberty and menopause to some extent by their relatively high gonadal estrogen production during this time. Then, around age 45, several years before menopause sets in at a mean age of 51.4 years, estrogen production begins to fall [19]. Thus women would lose the protection estrogens give, which could account for their second peak of illness onset after age 45.

A number of risk factors appear to counteract the protective effect of estrogens. Thus, the sex difference in the age of onset diminishes in the subgroup of cases with a genetic risk and in patients with perinatal complications [20-21].

Recent results regarding the age of menarche further support the hypothesis that physiological estrogens play a protective role against the development of the disease. We demonstrated a significantly later age of menarche in a representative group of first admitted women with schizophrenia and related psychoses as compared with a healthy control group [22]. Seeman and co-workers [23-24] found that later menarche was associated with an earlier onset of the illness, an association that was independent of factors such as family history and obstetric complications.

\subsection{Clinical findings}

Clinically, psychotic symptomatology has often been found to correlate with the estrogenic state of women (for review [1, 25]. For example, during high estrogen phases such as pregnancy chronic psychoses appear to improve, whereas there is an excess of psychoses after delivery.

Psychosis associated with estrogen withdrawal due to conditions other than the puerperium was recently reviewed by Mahé and Dumaine [26]. Those investigators reported cases of premenstrual psychosis, post abortion psychosis, and psychoses associated with removal of hydatiform mole, cessation of oral contraceptives, clomiphene and tamoxifen administration (both estrogen receptor 
antagonists), and gonadorelin agonist administration (which blocks pituitary stimulation of endogenous estrogen secretion). Psychotic episodes were acute, short and with a wide range of psychotic, but also affective, symptomatology. Recurrences were often reported when estrogen levels were normalized, and puerperal psychosis was frequent in the history of patients who were affected.

Psychotic symptoms in schizophrenic patients have also often been shown to deteriorate premenstrually or perimenstrually (i.e. in the low estrogen phase of the cycle; for review [1, 22, 25]. Thus, Riecher-Rössler et al. [27-28] could show an inverse correlation of estradiol blood levels with psychopathology. They examined 32 acutely admitted women with schizophrenia, who gave a history of regular menstrual cycles, and found a significant excess of admissions during the perimenstrual low estrogen phase of the cycle $(p<0.005)$. During the hospital stay of the 32 women, there emerged a significant association between estradiol levels on the one hand and psychopathology scores on the other: Psychopathology seemed to improve when estradiol blood levels rose and vice versa. This was not only true for the total score of the Brief Psychiatric Rating Scale (BPRS [29]) and for almost all the subscores of this scale like anergia, thought disturbance, activation and hostile suspiciousness, but also for the general behaviour on ward as rated by the nurses (NOSIE [30]) and for general well-being and paranoid feelings as rated by the patients themselves (BfS and paranoid subscore of PDS, both by von Zerssen and Koeller [31]).

Also, Bergemann et al. [32] found a significant effect of the menstrual cycle phase and $17-\beta$-estradiol levels on positive and negative symptoms of 125 women with schizophrenia and related psychoses. The same authors [33] could furthermore show a significant effect of estrogen on the comprehension of metaphoric speech and/or concretism, a main feature of schizophrenic thought and language disturbance. Ko et al. [34] similarly found that 35 women with chronic schizophrenia and related psychoses had low levels of estrogen which was associated with severe negative symptomatology and reduced cognitive functioning, especially in the domains of verbal performance and executive tasks.

Most studies, however, did examine the direct correlation between psychosis symptomatology and estradiol serum levels directly. For example, a more recent study [35], reported behavioural, affective and somatic symptoms of schizophrenia (not psychotic ones) to be associated with the menstrual cycle phase. Estradiol was not measured.

Rather than examining cyclic fluctuations, Hoff et al. [36] assessed the relationship between average estrogen levels from 4 consecutive weeks sampling with psychopathology and cognitive functioning in 22 female inpatients (aged between 22 and 63 years) with chronic schizophrenia. There was no significant association between average estrogen levels and psychopathology, but higher average estrogen levels were strongly associated with better cognitive abilities. However, this finding may be due in part to the effects of ageing.

It has to be noted that an elevated number of admissions during the perimenstrual period has also been identified in other disorders [37], and exacerbation of 
many psychiatric symptoms (not only psychotic ones) during the perimenstrual period was observed in patients with schizophrenia and related psychoses [27, 38]. In theory, this lack of specificity is to be expected because of the multiple effects of estrogen on mental functioning.

Studies on gender differences conducted in the area of late-onset schizophrenia emphasise the significance of hormone changes related to the menopause. Reicher and colleagues $[15,22]$ showed that there are twice as many women as men with the onset of schizophrenia and related psychoses beyond 40 years of age, and that female patients with late onset illness suffered from unexpectedly severe disease in terms of symptomatology and course. One explanation for this could be the fluctuation and sharp decline in estrogen levels just before and during the menopause. In support of this proposed explanation are results from long-term studies in women with schizophrenia showing that the course of illness in women tends to deteriorate rapidly during menopause and thereafter (for review [1, 39].

\subsection{Intervention studies}

Intervention studies have also been conducted over long periods, mainly with positive results [40-41] (for review [1]). Thus, as early as the 1940s, Manfred Bleuler [42] reported the first unsystematic trials using a combination of ovarian and pituitary hormones. Mall [43], a German psychiatrist in charge of a large hospital, examined 167 women suffering from schizophrenia with respect to estrogen excretion in 24-h urine samples, basal temperature and vaginal cytology. Based on his findings, he divided the psychoses into two groups: hypofollicular and hyperfollicular. In the former group, he replaced estrogens and found that "hypofollicular psychosis can be healed relatively easily by this substitution therapy". Unfortunately, Mall does not give many details about these interesting studies.

In the first systematic trial conducted in 1996, Kulkarni et al. [44] found that women with schizophrenia receiving estradiol as an adjunct to antipsychotic medication treatment exhibited rapid and greater improvement in psychotic symptoms than women receiving antipsychotics alone. In 2001, the same group performed a double-blind, 28-day, placebo-controlled study [45] in which 12 women were administered transdermal 17-ß-estradiol (patches) 50 microgram/24 h, another 12 women received 100 microgram $/ 24 \mathrm{~h}$ patches, and the third group received placebo patches. The 100 microgram group experienced greater improvement than either the 50 microgram or placebo groups; with striking improvements observed in the key psychotic symptoms.

In 2003, Akhondzadeh et al. [46] published a randomized study of 32 women of childbearing age with chronic schizophrenia. They administered ethinyl estradiol as an adjunct to haloperidol over 8 weeks while the control group received haloperidol only. The combination with estradiol showed a significant superiority over haloperidol alone regarding positive and negative symptom response. Fur- 
thermore, the estrogen group needed significantly less adjunctive anticholinergic medication to treat extrapyramidal side effects caused by haloperidol treatment. This finding is in line with other studies suggesting that estrogen treatment can also reduce the severity of antipsychotic medication-induced extrapyramidal side effects ([47]).

Louza et al. 2004 [48] did not find a positive response to estrogen treatment in their study, which they correctly discussed as possibly being due to having used conjugated estrogens rather than 17-ß-estradiol. In a Cochrane review in 2005, Chua et al. [49] surveyed data from only five randomised double blind intervention studies with appropriate methodology. They concluded that the effects of estrogen as sole treatment or adjunctive therapy for those with schizophrenia and related psychoses were still unclear, but that further larger, clinical trials were needed. Studies in this review with negative results used conjugated estrogens and not $17-\beta$-estradiol, although the latter has been shown to be the estrogen type with the most potent activity in the brain. Furthermore, in order to prevent endometrial hyperplasia, the estrogens were usually combined with progestogens which can counteract the positive effects of estradiol in the brain.

Kulkarni et al. [50] recently conducted a proof-of-concept study of 102 women with DSM-IV schizophrenia. In this double-blind randomized controlled 28-day study, women received either an active $100 \mu$ g estradiol skin patch treatment $(n=56)$ or an identical placebo patch $(n=46)$. All patients received antipsychotic drug treatment according to a standardized protocol. Progesterone was not given during the study, hence the trial measured the impact of unopposed estradiol. Psychopathology was assessed using the PANSS rating scale. Serum levels of estrogen, progesterone, prolactin, luteinising hormone, and follicle stimulating hormone were measured. Several cognitive tests were also administered. They found that patients who received the $100 \mu \mathrm{g}$ estradiol adjunct made a significantly better recovery in their total positive, negative, and general symptoms of schizophrenia than the patients who received standard antipsychotic medication only $(\mathrm{P}<0.01)$. Women who received the estradiol patch also showed significant improvement in cognition $(\mathrm{P}<0.01)$. By measuring luteinising hormone, they could also demonstrate that there is a direct effect on the pituitary gland, which suggests that this dose and type of unconjugated estrogen directly affects the hypothalamicpituitary-gonadal axis.

Overall, these studies provide strong evidence for the estrogen protection hypothesis. The addition of transdermally delivered estradiol seems to be associated with significant abatement of psychotic symptoms in women with schizophrenia compared with standardized antipsychotic drug treatment alone.

Most of the estrogen treatment studies conducted so far have been in young, reproductive age women and not in peri- or postmenopausal women with estrogen deficiency. Theoretically, the greatest effect of estradiol would be expected when it is replaced in a woman in a hypoestrogenic state. Good [51] conducted a study in postmenopausal patients. He administered estradiol and progesterone to 14 
women with schizophrenia, schizophreniform disorder or schizoaffective disorder and found a significant improvement of negative symptoms over six months.

There are also some case reports regarding positive results of hormone replacement therapy (HRT) in postmenopausal women with schizophrenia and related psychoses. Bergemann et al. [52] reported a case study of a woman with first onset of schizophrenia in the perimenopause period. The patient experienced severe acute psychosis symptoms over several months, but refused antipsychotic treatment. As she was diagnosed to be in a periclimacteric state based on clinical symptoms and hormone analysis, she was started on transdermal estradiol in combination with norethisterone acetate and had an impressive remission of the psychotic symptoms.

Lindamer [41] reported details about a postmenopausal woman, whose psychotic symptoms improved with estradiol treatment as an adjunct to her antipsychotic.

In 2001, Lindamer and associates [53] studied a community sample of postmenopausal women with schizophrenia and related psychoses. Twenty-four women received standard hormone replacement treatment (HRT), twenty-eight women had never received hormone treatment. Interestingly, the users of HRT needed a relatively lower average dose of antipsychotic medication and suffered fewer severe negative symptoms.

Ahokas et al. [54] described positive effects of estrogen treatment in women with postpartum psychosis. In those women who exhibited sustained estrogen deficiency states, the addition of 17-ß-estradiol, without any further medication, yielded a dramatic antipsychotic effect within 1 week. However, the proportion of schizophrenia-like psychoses in the sample was not given.

Finally, Kulkarni et al. [55] also tested the use of adjunctive estradiol in a small sample of 11 men with schizophrenia. They gave $2 \mathrm{mg}$ oral estradiol valerate as an adjunct to six men who were taking antipsychotic drugs. Five men received oral placebo plus their standard antipsychotic medication for 7 days. This small study was only conducted for 7 days to avoid feminisation and other side effects in the men. The groups were matched for age, illness severity and duration. Oral, rather than transdermal, estradiol was used to ensure treatment adherence in men with acute psychosis. Psychopathology was assessed using the standardized rating scales, PANSS, and the Brief Psychiatric Rating Scale, BPRS. By day 5, the estradiol group showed significant abatement of psychotic symptoms compared with the placebo group and, by day 7, the estradiol group made further improvements. This study, although small in sample size and short in duration, raises the possibility that non-feminising estrogen may provide useful treatment possibilities for men with schizophrenia. 


\section{Hypo-estrogenism in women with schizophrenia (The hypothesis of hypoestrogenism)}

Several studies have recently confirmed earlier findings of disturbed gonadal function and hypo-estrogenism in women with schizophrenia [1, 27, 35, 39, 44, 56-61]. They described menstrual irregularities and reduced blood levels of estradiol, progesterone and gonadotropins (follicle-stimulating hormone, luteinizing hormone) throughout the menstrual cycle, plus anovulation in the majority of women with schizophrenia and related psychoses. Reduced fertility was also reported.

There appear to be multiple reasons for these disturbances including the consequences of emotional stress and/or antipsychotic medication induced hyperprolactinaemia, which is known to suppress gonadal function [62]. However, these are probably not the only causes, because women experiencing other psychiatric disorders with similar emotional stress do not have the same hypothalamic - pituitary - gonadal axis hormone changes, at least not to the same degree [39, 59]. Furthermore, hypoestrogenism was observed long before the introduction of antipsychotics.

Smith et al. [60] found the dose of typical antipsychotics to correlate with prolactin levels especially in women and prolactin to correlate inversely with estradiol serum levels. In contrast to those findings, Huber et al. [59] were unable to identify a significant association of prolactin and estradiol in 43 women with acute psychosis, 14 women with other diagnoses and 9 healthy control women. Nevertheless, the women with schizophrenia and related psychoses had significantly lower estradiol serum levels than the control women. Women with other psychiatric diagnoses fell in between the psychotic and the healthy group with regard to estradiol and prolactin levels. Also, Canuso et al. [57] found a high rate of ovarian dysfunction and estradiol levels below normal, irrespective of medication type or prolactin status in 16 premenopausal women with schizophrenia and schizoaffective disorders,. Interestingly, Warner et al. [63] found prolactin levels in unmedicated schizophrenic patients to be even lower than in control individuals. Those investigators suggested that this was due to a disordered dopaminergic system because dopamine tonically inhibits prolactin.

Taken together, these results imply that the hypothalamic-pituitary-gonadal axis is disturbed in many women with schizophrenia and related psychoses, and that the reasons for this are far from clear yet. An interesting research question in this context is whether gonadal dysfunction with estrogen deficiency could even be part of the underlying pathogenetic process, at least in a subgroup of women [22]. 


\section{$4 \quad$ Implications for clinicians and researchers}

Further research into the impact of gonadal function and estrogen on schizophrenia and related psychoses is warranted because new diagnostic and therapeutic strategies could emerge that would benefit the many women worldwide who suffer from this disorder.

\subsection{Assessment and therapy of gonadal dysfunction}

As there is growing evidence that many even younger women with schizophrenia and related psychoses are in a state of estrogen deficiency, in the future estrogens and the gonadal axis should be considered more seriously in the treatment of women with schizophrenia and related psychoses. Psychiatric history taking should always include questions regarding menstrual irregularities, amenorrhoea and galactorrhoea. Also, prolactin and estrogen serum levels should be tested, if necessary. Gonadal dysfunction and hypoestrogenic states can often be found even in menstruating women [27, 39, 60]. In addition, hyperprolactinaemia is clearly under-diagnosed [62]. Some authors have therefore suggested routine laboratory tests [60].

Most antipsychotics can cause hyperprolactinaemia and- especially if they are taken over a number of years - theoretically induce 'iatrogenic early menopause' via suppression of physiological estradiol production. The concomitant risks include both short-term effects, such as hot flushes and sexual dysfunction, as well as long-term consequences, including osteoporosis and potentially cardiovascular disease or cognitive deterioration [4, 62]. In patients with schizophrenia, these risks are further increased by additional risk factors such as smoking, poor diet and reduced exercise [60]. Furthermore, menopausal complaints may lead to compliance problems.

In the case of hyperprolactinaemia with secondary estrogen deficiency, prolactin-sparing antipsychotics (e.g. clozapine, quetiapine, aripiprazole or maybe olanzapine [62] should therefore be preferred. If a switch to these antipsychotics is not possible for clinical reasons or if hypoestrogenism persists despite switching, then estrogen can be added to the treatment. Issues regarding contraception must be taken into account in such cases because, when switching to prolactin-sparing antipsychotics, the menstrual cycle often normalizes and fertility is regained, with high risk for unplanned pregnancy [64]. 


\subsection{Estradiol as a therapeutic agent?}

First trials of estrogens in schizophrenia and related psychoses indicate that estradiol could be used as an adjunct to antipsychotic medication. However, further replications of these findings in larger control studies by different groups are needed before recommendations for broad clinical application can be made.

In women who suffer from frequent perimenstrual psychotic relapses, 'cycle modulated' antipsychotic medication therapy or, if contraception is needed at the same time, continuous use of oral contraceptives without hormone-free intervals may be strategies worthy of research $[22,65]$.

Even more promising could be hormonal replacement with estrogens in women with schizophrenia in peri- and postmenopause, because estrogens in other disorders such as depression have proven to be especially helpful when they are used to restore hormonal balance.

Hormonal replacement with estrogens for women with schizophrenia during and after the perimenopause could be recommended as an augmentation strategy respectively an adjunct to antipsychotic medication. Possibly, the dose of antipsychotics could then be reduced and corresponding side-effects minimized. The replacement of estrogens in these women could also attenuate perimenopausal complaints such as hot flushes, night sweats with sleep-disturbances and general irritability (see Table 11.1) which can contribute to a general deterioration of the mental state and, in vulnerable women, potentially provoke a psychotic episode. In contrast to this recommendation, it has been reported that women with schizophrenia are less likely to ever use HRT as compared to women without psychiatric diagnoses [66].

Estrogen replacement therapy for women of this age group has been recommended anyway for many reasons, for example prophylaxis of osteoporosis, and also delay of age-dependent cognitive deterioration or Alzheimer's dementia [67] (for review see [68]; (Table 11.1). Further research into estrogen treatment as an additional indication in peri- and postmenopausal women with schizophrenia and related psychoses is urgently needed.

\section{Insert Table 11.1 here}

The use of estrogen has been questioned in the context of perimenopausal estrogen replacement by studies such as the WHI, Women's Health Initiative Study [69], the WHI-M, Women's Health Initiative Memory Study [70] and the HERS, Heart and Estrogen/Progestin Replacement Study [71]. These studies have highlighted the side effects of hormone replacement therapy which has provoked an ongoing controversy about the advantages and disadvantages of this regime.

However, the WHI study has been criticised by many experts and by the International Menopause Society [72] because of the advanced age of the study population (mean age at inclusion was 63 years) who had a high prevalence of cardiovas- 
cular risk factors. Many of the complications noted for participants in the WHI study such as stroke, pulmonary embolism and myocardial infarction which were attributed to the vascular effects of estrogens, could well have been due to preexisting arteriosclerosis.

The WHI conclusions have now been partially counteracted by a reanalysis [73], which showed that the cardiovascular complications can be reduced by using replacement therapy early in the perimenopause. This has opened a window of opportunity in which possibly even a cardiovascular benefit can be obtained in healthy menopausal women when replacement therapy is started early after the menopause (review [68]).

Overall, the use of estrogens for therapeutic reasons must be distinguished from their preventative use. The WHI investigated the latter use and as described above, concerns about the conclusions have been made. The potential therapeutic use in women with psychosis or other mental disorders has been discussed here, and while care must be given to monitoring potential side -effects, the benefits for women with poor quality of life due to intractable schizophrenia often outweigh the risk of side- effects.

Further research needs to be carried out into the best mode of HRT for psychiatric patients. To date, the natural 17- $\beta$-estradiol has been shown to have the best neuropsychoprotective effects compared with other estrogens that are often prescribed. Transdermal application in the form of patches or gel is preferred because of smoother metabolism and potentially fewer side effects. Progestogens are usually added to estrogens in order to prevent endometrial cancer, but they can antagonize the positive effects of estrogens with respect to mental state [2, 65]. In addition, the systemic effects of progestogens can be minimized, potentially by other forms of application such as via an intrauterine device (IUD).

Furthermore, alternatives to conventional HRT, i.e. compounds with more specific and potent estrogenic activity in the brain as opposed to other tissues, need to be investigated $[22,75]$. Such compounds would both minimize the side effects of hormonal therapy and permit new therapeutic strategies in men. Possible candidates are selective estrogen receptor modulators, which have agonistic or antagonistic properties that depend on the target tissue. However, the effects of the available selective estrogen receptor modulators on the brain remain to be clarified. Raloxifene, for example, appears to exert its main effects on the bone, although recent data suggest that it also acts on different brain receptors [70]. A recent study by Kulkarni et al has shown promising antipsychotic effects for adjunctive raloxifene in postmenopausal women with schizophrenia [78]. Also, the synthetic steroid tibolone appears to cause less endometrial proliferation, but its effects on the central nervous system are still not clear, apart from the fact that it appears to have an androgenic effect and increases $\beta$-endorphin levels, with improvement in mood and libido [76]. Further studies on the brain-specific effects of selective estrogen receptor modulators and other estrogenic compounds (e.g. phyto-estrogens, xeno-estrogens and dihydroepiandrosterone) are urgently needed. 


\section{Summary and conclusions}

In summary, there is emerging evidence that estrogens are very useful neuroprotective and psychoprotective adjunctive therapies, which complement and enhance the traditional drug therapies for people with schizophrenia and related psychoses. However, it must be emphasized that the role for estrogen treatment strategies still requires further research. In particular, results from large, control studies are needed before estrogens may be recommended as adjunct therapy in standard clinical practice for the treatment of women with schizophrenia, without proven estrogen deficiency. In contrast, other strategies should already be part of standard clinical care [77]. These include examination of the gonadal axis, with therapeutic actions, if indicated. The decision to use estrogen replacement therapy, must always be made on the basis of an individual risk-benefit assessment (NAMS 2000; Writing Group for the Women's Health Initiative Investigators 2002) and in close collaboration with a gynaecologist.

For future research, many questions remain unresolved, not only regarding new therapeutic strategies and compounds but also regarding the poorly understood disturbances of estrogens and the hypothalamic-pituitary-gonadal axis in women with schizophrenia and related psychoses. Further research in this area may substantially contribute to our understanding of the pathogenesis of this disease, at least in a subgroup of women. 


\section{References}

1. Riecher-Rössler A, Häfner H (1993) Schizophrenia and oestrogens - is there an association? Eur Arch Psychiatry Clin Neurosci 242:323-8

2. Cyr M, Calon F, Morissette M, Di Paolo T (2002) Estrogenic modulation of brain activity: implications for schizophrenia and Parkinson's disease. J Psychiatry Neurosci 27:12-27

3. Goldstein JM, Seidman LJ, O'Brien LM, Horton NJ, Kennedy DN, Makris N, et al. (2002) Impact of normal sexual dimorphisms on sex differences in structural brain abnormalities in schizophrenia assessed by magnetic resonance imaging. Arch Gen Psychiatry 59:154-64

4. Oesterlund M (2002) The role of estrogens in neuropsychiatric disorders. Curr Opin Psychiatry 15:307-12

5. Vedder H, Behl C. Estrogens in neuropsychiatric disorders: from physiology to pathophysiology. In: Bergemann N, Riecher-Rössler A, editors. Estrogen effects in psychiatric disorders. Wien, New York: Springer; 2005. p. 1-30.

6. Garcia-Segura L, Azcoitia I, Doncarlos L (2001) Neuroprotection by estradiol. Prog Neurobiol 63:29-60

7. McEwen B (2002) Interplay between membrane and genomic actions of estrogens. Int J Neuropsychopharmacol 5 (Suppl 1):12

8. Stahl SM (2001) Effects of estrogen on the central nervous system. J Clin Psychiatry 62:317-8

9. Stahl SM (2001) Why drugs and hormones may interact in psychiatric disorders. J Clin Psychiatry 62:225-6

10. Balthazart J, Ball GF (2006) Is brain estradiol a hormone or a neurotransmitter? Trends Neurosci 29:241-9

11. Oesterlund MK, Keller E, Hurd YL (2000) The human forebrain has discrete estrogen receptor alpha messenger RNA expression: high levels in the amygdaloid complex. Neuroscience 95:33342

12. Weickert CS, Miranda-Angulo AL, Wong J, Perlman WR, Ward SE, Radhakrishna V, et al. (2008) Variants in the estrogen receptor alpha gene and its mRNA contribute to risk for schizophrenia. Hum Mol Genet 17:2293-309

13. Yonkers KA, Kando JC, Cole JO, Blumenthal S (1992) Gender differences in pharmacokinetics and pharmacodynamics of psychotropic medication. Am J Psychiatry 149:587-95

14. Häfner H, Riecher-Rössler A, An Der Heiden W, Maurer K, Fatkenheuer B, Löffler W (1993) Generating and testing a causal explanation of the gender difference in age at first onset of schizophrenia. Psychol Med 23:925-40

15. Riecher-Rössler A, Löffler W, Munk-Jorgensen P (1997) What do we really know about lateonset schizophrenia? Eur Arch Psychiatry Clin Neurosci 247:195-208

16. Riecher A, Maurer K, Löffler W, Fätkenheuer B, An Der Heiden W, Munk-Jorgensen P, et al. Gender differences in age at onset and course of schizophrenic disorders. In: Häfner H, Gattaz W, editors. Search for the causes of schizophrenia. Berlin, Heidelberg: Springer; 1991. p. 14-33.

17. Häfner H, Riecher-Rössler A, Hambrecht M, Maurer K, Meissner S, Schmidtke A, et al. (1991) Geschlechtsunterschiede bei schizophrenen Erkrankungen. Fortschr Neurol Psychiatr 59:343-60

18. Häfner H, Behrens S, De Vry J, Gattaz WF, Löffler W, Maurer K, et al. (1991) Warum erkranken Frauen später an Schizophrenie? Erhöhung der Vulnerabilitätsschwelle durch Östrogen. Nervenheilkunde 10:154-63

19. Labhart A (1978) Klinik der inneren Sekretion. Berlin, Heidelberg, New York, Springer

20. Häfner H. Gender differences in schizophrenia. In: Bergemann N, Riecher-Rössler A, editors. Estrogen effects in psychiatric disorders. Wien, New York: Springer; 2005. p. 53-94.

21. Könnecke R, Häfner H, Maurer K, Löffler W, an der Heiden W (2000) Main risk factors for schizophrenia: increased familial loading and pre- and peri-natal complications antagonize the protective effect of oestrogen in women. Schizophr Res 44:81-93

22. Riecher-Rössler A (2002) Oestrogen effects in schizophrenia and their potential therapeutic implications--review. Arch Womens Ment Health 5:111-8 
23. Cohen RZ, Seeman MV, Gotowiec A, Kopala L (1999) Earlier puberty as a predictor of later onset of schizophrenia in women. Am J Psychiatry 156:1059-64

24. Hayeems R, Seeman MV. Puberty and schizophrenia onset. In: Bergemann N, Riecher-Rössler A, editors. Estrogen efects in psychiatric disorders. Wien, New York: Springer; 2005. p. 95.

25. Seeman MV (1996) Schizophrenia, gender, and affect. Can J Psychiatry 41:263-4

26. Mahe V, Dumaine A (2001) Oestrogen withdrawal associated psychoses. Acta Psychiatr Scand 104:323-31

27. Riecher-Rössler A, Häfner H, Stumbaum M, Maurer K, Schmidt R (1994) Can estradiol modulate schizophrenic symptomatology? Schizophr Bull 20:203-14

28. Riecher-Rössler A, Häfner H, Dutsch-Strobel A, Oster M, Stumbaum M, van Gulick-Bailer M, et al. (1994) Further evidence for a specific role of estradiol in schizophrenia? Biol Psychiatry 36:492-4

29. Overall J, Gorham D (1962) The brief psychiatric rating scale. Psychol Report 10:799-812

30. Honigfeld G, Gillis R, Klett C, editors. NOSIE: Nurses' observation scale for inpatient evaluation. Rockville, MD: NIMH; 1976.

31. von Zerssen D, Koeller D (1976) Klinische Selbstbeurteilungs-Skalen (KSb-Si) aus dem Münchener Psychiatrischen Informations-System (PSYCHIS München). Manual. Weinheim, Beltz

32. Bergemann N, Parzer P, Runnebaum B, Resch F, Mundt C (2007) Estrogen, menstrual cycle phases, and psychopathology in women suffering from schizophrenia. Psychol Med 37:1427-36

33. Bergemann N, Parzer P, Jaggy S, Auler B, Mundt C, Maier-Braunleder S (2008) Estrogen and comprehension of metaphoric speech in women suffering from schizophrenia: results of a doubleblind, placebo-controlled trial. Schizophr Bull 34:1172-81

34. Ko YH, Joe SH, Cho W, Park JH, Lee JJ, Jung IK, et al. (2006) Estrogen, cognitive function and negative symptoms in female schizophrenia. Neuropsychobiology 53:169-75

35. Choi SH, Kang SB, Joe SH (2001) Changes in premenstrual symptoms in women with schizophrenia: a prospective study. Psychosom Med 63:822-9

36. Hoff AL, Harris D, Faustman WO, Beal M, DeVilliers D, Mone RD, et al. (1996) A neuropsychological study of early onset schizophrenia. Schizophr Res 20:21-8

37. Althaus G, Pfuhlmann B, Franzek E (2000) [The effect of the menstruation cycle on manifestations of pychiatric diseases]. Fortschr Neurol Psychiatr 68:357-62

38. Harris AH (1997) Menstrually related symptom changes in women with schizophrenia. Schizophr Res 27:93-9

39. Riecher-Rössler A, Häfner H, Dütsch-Strobel A, Stumbaum M (1998) Gonadal function and its influence on psychopathology. A comparison of schizophrenic and non-schizophrenic female inpatients. Arch Womens Ment Health 1:15-26

40. Korhonen S, Saarijarvi S, Aito M (1995) Successful estradiol treatment of psychotic symptoms in the premenstrual phase: a case report. Acta Psychiatr Scand 92:237-8

41. Lindamer LA, Lohr JB, Harris MJ, Jeste DV (1997) Gender, estrogen, and schizophrenia. Psychopharmacol Bull 33:221-8

42. Bleuler M (1943) Die spätschizophrenen Krankheitsbilder. Neurology 15:259-90

43. Mall G (1960) Diagnostik und Therapie ovarieller Psychosen. Zentralblatt für die gesamte Neurologie und Psychiatrie 155:250

44. Kulkarni J, de Castella A, Smith D, Taffe J, Keks N, Copolov D (1996) A clinical trial of the effects of estrogen in acutely psychotic women. Schizophr Res 20:247-52

45. Kulkarni J, Riedel A, de Castella AR, Fitzgerald PB, Rolfe TJ, Taffe J, et al. (2001) Estrogen - a potential treatment for schizophrenia. Schizophr Res 48:137-44

46. Akhondzadeh S, Nejatisafa AA, Amini H, Mohammadi MR, Larijani B, Kashani L, et al. (2003) Adjunctive estrogen treatment in women with chronic schizophrenia: a double-blind, randomized, and placebo-controlled trial. Prog Neuropsychopharmacol Biol Psychiatry 27:1007-12

47. Thompson KN, Kulkarni J, Sergejew AA (2000) Extrapyramidal symptoms and oestrogen. Acta Psychiatr Scand 101:130-4 
48. Louza MR, Marques AP, Elkis H, Bassitt D, Diegoli M, Gattaz WF (2004) Conjugated estrogens as adjuvant therapy in the treatment of acute schizophrenia: a double-blind study. Schizophr Res 66:97-100

49. Chua W, De Izquierdo S, Kulkarni J, Mortimer AM (2005) Estrogen for schizophrenia. The Cochrane database of systematic reviews, 4: 10.1002/14651858.CD0044719.pub2

50. Kulkarni J (2009) Oestrogen--a new treatment approach for schizophrenia? Med J Aust 190:S37-8

51. Good KP, Kopala LC, Martzke JS, Fluker M, Seeman MV, Parish B, et al. (1999) Hormone replacement therapy in postmenopausal women with schizophrenia: preliminary findings. Schizophr Res 12:131

52. Bergemann N, Abu-Tair F, Strowitzki T (2007) Estrogen in the treatment of late-onset schizophrenia. J Clin Psychopharmacol 27:718-20

53. Lindamer LA, Buse DC, Lohr JB, Jeste DV (2001) Hormone replacement therapy in postmenopausal women with schizophrenia: positive effect on negative symptoms? Biol Psychiatry 49:4751

54. Ahokas A, Aito M, Rimon R (2000) Positive treatment effect of estradiol in postpartum psychosis: a pilot study. J Clin Psychiatry 61:166-9

55. Kulkarni J. Clinical estrogen trials in patients with schizophrenia. In: Bergemann N, RiecherRössler A, editors. Estrogen effects in psychiatric disorders. Vienna: Springer; 2005. p. 107-23.

56. Bergemann N, Parzer P, Nagl I, Salbach B, Runnebaum B, Mundt C, et al. (2002) Acute psychiatric admission and menstrual cycle phase in women with schizophrenia. Arch Womens Ment Health 5:119-26

57. Canuso CM, Goldstein JM, Wojcik J, Dawson R, Brandman D, Klibanski A, et al. (2002) Antipsychotic medication, prolactin elevation, and ovarian function in women with schizophrenia and schizoaffective disorder. Psychiatry Res 111:11-20

58. Hoff AL, Kremen WS, Wieneke MH, Lauriello J, Blankfeld HM, Faustman WO, et al. (2001) Association of estrogen levels with neuropsychological performance in women with schizophrenia. Am J Psychiatry 158:1134-9

59. Huber TJ, Rollnik J, Wilhelms J, von zur Muhlen A, Emrich HM, Schneider U (2001) Estradiol levels in psychotic disorders. Psychoneuroendocrinology 26:27-35

60. Smith S, Wheeler MJ, Murray R, O'Keane V (2002) The effects of antipsychotic-induced hyperprolactinaemia on the hypothalamic-pituitary-gonadal axis. J Clin Psychopharmacol 22:109-14

61. Zhang-Wong JH, Seeman MV (2002) Antipsychotic drugs, menstrual regularity and osteoporosis risk. Arch Womens Ment Health 5:93-8

62. Maguire GA (2002) Prolactin elevation with antipsychotic medications: mechanisms of action and clinical consequences. J Clin Psychiatry 63 Suppl 4:56-62

63. Warner MD, Walker AM, D'Souza DC, Lee D, Nasseri D, Peabody CA (2001) Lower prolactin bioactivity in unmedicated schizophrenic patients. Psychiatry Res 102:249-54

64. Neumann NU, Frasch K (2001) [Olanzapine and pregnancy. 2 case reports]. Nervenarzt 72:876-8

65. Braendle W, Breckwoldt M, Kuhl H (2001) Sexualhormone und Psyche - Ergebniosse des 2. Interdisziplinären Frankfurter Gesprächs zur Kontrazeption. Frauenarzt 42:154-60

66. Lindamer LA, Buse DC, Auslander L, Unutzer J, Bartels SJ, Jeste DV (2003) A comparison of gynecological variables and service use among older women with and without schizophrenia. Psychiatr Serv 54:902-4

67. Sherwin BB (2005) Estrogen and memory in women: how can we reconcile the findings? Horm Behav 47:371-5

68. Riecher-Rössler A, de Geyter C (2007) The forthcoming role of treatment with oestrogens in mental health. Swiss Med Wkly 137:565-72

69. Rossouw JE, Anderson GL, Prentice RL, LaCroix AZ, Kooperberg C, Stefanick ML, et al. (2002) Risks and benefits of estrogen plus progestin in healthy postmenopausal women: principal results From the Women's Health Initiative randomized controlled trial. JAMA 288:321-33

70. Craig MC, Maki PM, Murphy DG (2005) The Women's Health Initiative Memory Study: findings and implications for treatment. Lancet Neurol 4:190-4 
71. Hlatky MA, Boothroyd D, Vittinghoff E, Sharp P, Whooley MA (2002) Quality-of-life and depressive symptoms in postmenopausal women after receiving hormone therapy: results from the Heart and Estrogen/Progestin Replacement Study (HERS) trial. JAMA 287:591-7

72. Birkhäuser MH, Panay N, Archer DF, Barlow D, Burger H, Gambacciani M, et al. (2008) Updated practical recommendations for hormone replacement therapy in the peri- and postmenopause. Climacteric 11:108-23

73. Rossouw JE, Prentice RL, Manson JE, Wu L, Barad D, Barnabei VM, et al. (2007) Postmenopausal hormone therapy and risk of cardiovascular disease by age and years since menopause. JAMA 297:1465-77

74. Pines A, Sturdee DW, Birkhauser MH, Schneider HP, Gambacciani M, Panay N (2007) IMS updated recommendations on postmenopausal hormone therapy. Climacteric 10:181-94

75. Halbreich U (2002) The spectrum of estrogens, estrogen agonists and serms. Int J Neuropsychopharmacol 5 (Suppl 1):12

76. Davis SR (2002) The effects of tibolone on mood and libido. Menopause 9:162-70

77. Grigoriadis S, Seeman MV (2002) The role of estrogen in schizophrenia: implications for schizophrenia practice guidelines for women. Can J Psychiatry 47:437-42

78 Kulkarni J, Gurvich C, Lee S,Gilbert H,Gavrilidis E, de Castella A,Berk M, Dodd S, Fitzgerald P,Davis SR (2010) Piloting the effective dose of adjunctive selective estrogen receptor modulator in postmenopausal women with schizophrenia. Psychoneuroendocrinology Feb 18 (E Pub ahead of print) 


\section{Table}

\section{1}

\section{Some}

im-

\section{portant}

\section{effects}

of estro-

\section{gen re-}

\section{place-}

\section{ment}

Positive

Perimenopausal complaints $\downarrow$

physical: hot flushes, genital discomfort, aging of collagen (skin, joints, intervertebral discs) $\downarrow$ ? mental: depression, irritability, emotional lability $\downarrow$

Risk of osteoporosis $\downarrow$

Delay of cognitive decline/Morbus Alzheimer?

Cardiovascular protection?

(if started right after menopause)
Negative

Endometrial carcinoma $\uparrow$ if unopposed estrogens are administered

$(\rightarrow$ in women without hysterectomy always combine with progestogens!)

Risk of breast cancer $\uparrow$ ?

$(\rightarrow$ not in patients with a familiar or own risk and usually not longer than 7 years!)

Risk of thrombosis and cerebral insult $\uparrow$ ? $(\rightarrow$ no prescription for patients at risk!)

Other cardiovascular risks (coronary heart disease, arteriosclerosis) $\uparrow$ ?

$(\rightarrow$ start only within the first 10 years after menopause and not in patients with cardiovascular disease!)

Sources: [68, 72-73] 